\title{
The Hedgehog and the hair follicle: a growing relationship
}

\author{
Andrzej Dlugosz
}

Department of Dermatology and Comprehensive Cancer Center, University of Michigan, 3310 CCGC/Box 0932, 1500 E. Medical Center Drive, Ann Arbor, Michigan 48109-0932, USA Phone: (734) 763-0355; Fax: (734) 763-4575; E-mail: dlugosza@umich.edu

The various forms of hair loss (alopecia) constitute an extremely common complaint among both men and women. Although sometimes dismissed as merely cosmetic, these conditions can be psychologically and socially devastating. The desire to regrow hair is at the root of a multi-billion dollar industry worldwide; however, there are few truly effective treatments. Given the recent progress in elucidating some of the signals regulating hair follicle growth and development, there is hope that more useful therapies may soon be available. The timely report by Sato et al. (1) presents interesting new data bearing on this issue.

The mature hair follicle is constructed of concentric layers of epithelial cells surrounding the hair shaft (Figure 1). The deepest portion of the follicle, the hair bulb, contains a mesenchymal dermal papilla enveloped by rapidly proliferating hair matrix cells. These epithelial cells give rise to 6 terminally differentiated cell types in the hair shaft and inner root sheath. Most hairs contain a medulla, cortex, and cuticle; external to this are the inner root sheath cuticle, and Huxley's and Henle's layers. The outer root sheath is continuous with the epidermis and sebaceous gland, and forms the outermost layer, extending down to and surrounding the hair follicle bulb. A specialized region of the outer root sheath called the "bulge," located just beneath the sebaceous gland at the insertion site of the arrector pili muscle, contains cells with stem cell properties $(2,3)$. Actively growing follicles also contain melanocytes that transfer pigment to matrix keratinocytes, imparting color to the developing hair. The follicle is thus a rich model for studying epithelial-mesenchymal interactions, cell lineage determination, melanogenesis, and the molecular regulation of growth and development.

Unlike other organs in the adult, the hair follicle is remarkably dynamic and continuously cycles through periods of active growth (anagen), regression (catagen), and quiescence (telogen) (4) (Figure 1). The duration of anagen is an important determinant of hair length, and may thus be as long as 6 years for scalp follicles and as short as 6-8 weeks for eyebrow follicles (5). In contrast to the complex anagen hair follicle, the telogen follicle is a diminutive organ consisting of a sebaceous gland, a small number of quiescent outer root sheath cells surrounding the base of the hair shaft, and dermal papilla cells lying adjacent to the remaining follicle epithelium. Activation of bulge keratinocytes in early anagen is believed to provide the transient amplifying cells needed to repopulate the newly forming follicle $(2,3)$. Hair cycling thus involves the programmed destruction and reconstruction of epithelial cells comprising the entire lower two thirds of the hair follicle, including the hair matrix and inner root sheath. Unlike the follicle epithelium, the number of dermal papilla cells remains relatively constant throughout the hair cycle. This small cluster of mesenchymal cells possesses remarkable inductive potential, and may hold the key to understanding how hair follicle growth is regulated: implantation of dermal papilla cells beneath naive epidermis results in de novo formation of mature hair follicles, even in skin that is normally hairless (6). As most nonscarring alopecias can be attributed to abnormalities involving the hair cycle, understanding how this process is regulated may yield important clues for effective treatment.
The reactivation of follicle growth with the onset of each anagen phase has led investigators to propose that some of the same signals that regulate follicle formation during embryogenesis may also operate in adult skin. The detailed analysis of classical tissue recombinants has revealed that the development of hair follicles and feathers, like that of other organs, is dependent on sequential inductive interactions involving adjacent epithelial and mesenchymal cell populations (7). Indeed, embryonic skin has served as an exceptionally useful experimental model for studying factors involved in patterning; the establishment and maintenance of polarity; growth; programmed cell death; and terminal differentiation (8).

As a foundation for understanding the molecular basis of hair follicle morphogenesis, the expression patterns of various genes involved in the development of other embryonic structures have been examined in skin. These genes encode members of the $F G F$, TGF- $\beta$, WNT, Hedgehog, Notch, and other signaling pathways (reviewed in refs. 9-11). The appearance of new hair follicles in postnatal mouse skin expressing a truncated $\beta$-catenin mutant (12) suggested an important role for this factor in the initiation of hair follicle development, although these mice also went on to develop well-differentiated hair follicle tumors.

Shh is one of a family of related ligands involved in a remarkable variety of developmental processes, including the establishment of left-right asymmetry, neural tube and somite patterning, craniofacial and limb development, and the formation of several organs (reviewed in refs. 13,14). Shh produced by one population of cells alters the developmental fate of neighboring target cells in a concentration-dependent manner. According to the current 


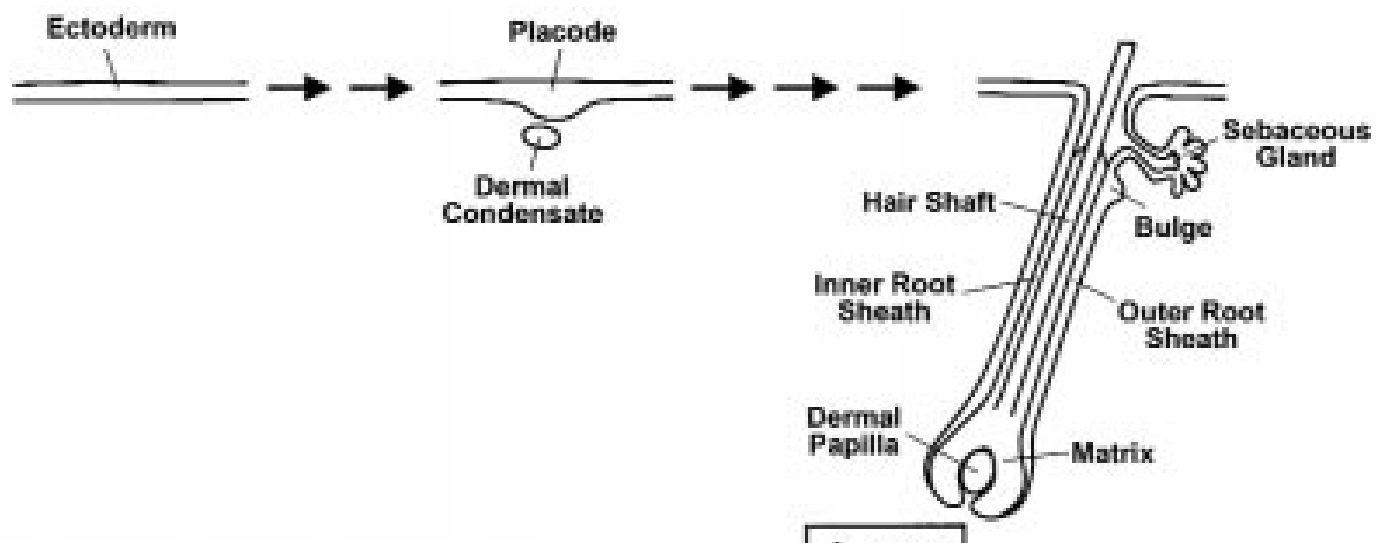

\section{Figure 1}

Hair follicle development and postnatal cycling. A series of epithelial and mesenchymal signals specify when and where primordial skin appendages, comprising an epithelial placode and dermal condensate, will appear. Additional signals bring about the rapid growth and tissue remodeling that underlie formation of a mature hair follicle, with Shh playing a pivotal role in this process. New findings suggest that Shh is also capable of operating in adult skin, where it may be involved in triggering reentry into the active growth phase of the hair cycle, (anagen).

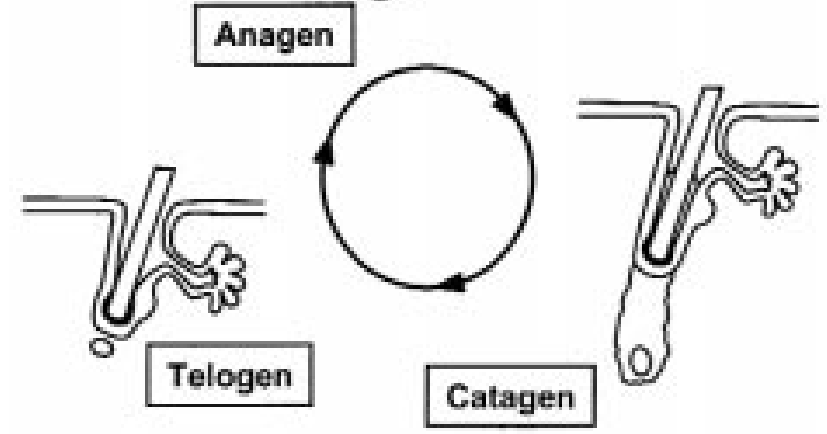

model, Shh interacts with and blocks the function of a cell-surface receptor called Ptch1, which normally inhibits a third protein called Smo (15). During embryogenesis, Shh-mediated derepression of Smo results in temporally and spatially restricted activation of Shh target genes, which include the transcription factor Gli1 and Ptch1 itself. The expression level of these transcripts is thus a reliable indicator of Shh pathway activity. Intense interest in Shh signaling in skin was fueled by the discovery that loss-of-function mutations affecting the $P T C H 1$ gene occur in both familial and sporadic basal cell carcinoma $(16,17)$, resulting in constitutive Shh pathway activation.

What is the normal function of Shh in skin? During development, Shh expression is restricted to the epithelial placode of primordial hair follicles, whereas Ptch1 mRNA is detected in both the placode and dermal condensate (Figure 1) $(18,19)$. This expression pattern suggested a role for Shh signaling in developing skin, and analysis of Shh mutant mice revealed that this molecule was required for hair follicle morphogenesis $(20,21)$. Although placodes and dermal condensates were detected in both wild-type and $S h h^{-/-}$ embryos, progression through subse- quent stages of hair follicle development was severely impaired in the skin of mutant animals. These studies revealed an essential function for Shh as a fundamental growth regulator in embryonic hair follicles, and set the stage for future studies exploring the potential involvement of Shh in postnatal follicle function.

In this issue, Sato et al. demonstrate that Shh, delivered by subcutaneous injection of an adenoviral expression vector, is capable of profoundly affecting the hair cycle in postnatal mice (1). They propose that Shh acts as a biologic switch that triggers telogen hair follicles to enter anagen. These investigators also demonstrate that spontaneous anagen is associated with transient upregulation of endogenous Shh and Ptch1 transcripts, suggesting that activation of the Shh pathway may be the physiological signal stimulating follicle growth. Although additional studies will be required to test this hypothesis, the data presented by Sato et al. convincingly show that transient expression of a single key regulatory molecule is sufficient to activate a complex morphogenetic program in postnatal rodent skin. Notably, the lack of any gross or histological abnormalities in skin 6 months after injection argues against any deleterious long-term consequences.

What is the specific target cell responding to exogenous Shh in injected skin, and is this same cell type activated by Shh during the normal transition to anagen? Based on the nature of the technique used for their studies, it is not surprising that Shh mRNA was detected in several different cell types scattered throughout the treated areas of skin (1). What is somewhat surprising is that the induction of anagen was remarkably uniform in the injected areas despite the fact that Shh expression was mosaic. To help understand this result, it will be important to identify the cells responding directly to Shh by examining the in situ expression of Shh target genes during the normal transition to anagen and in response to exogenous Shh. Even though the expression of exogenous Shh may be widespread, responsiveness of specific cell types to Shh in the skin is likely to be tightly regulated both in space and time (22). As there is strong evidence that dermal papilla cells play a central role in regulating growth of the follicle epithelium, perhaps these are the critical target cells for Shh, and the observed 
stimulation of anagen is mediated by diffusable keratinocyte mitogens secreted by this cell type.

Could somatic gene therapy for human hair loss become a reality? This, of course, is the multi-billion dollar question. Because the report by Sato et al. only illustrates the effectiveness of Shh in normal rodent skin, it is impossible to predict whether this molecule will have any utility in restoring hair growth in diseased human skin. Testing the effects of Shh in human scalp xenografts may be a reasonable next step. As for the prospect that modulation of the Shh pathway may provide a long sought after "cure" for androgenetic alopecia, it doesn't seem likely. This common condition is characterized by a progressive reduction in the duration of anagen and by miniaturization of hair follicles, with a gradual switch from the production of pigmented terminal hairs to unpigmented vellus hairs (23). Merely activating anagen without restoring a more normal follicle architecture is not likely to achieve the desired results. On the other hand, the increasing insight into signaling pathways regulating hair develop- ment will almost certainly yield additional therapeutic targets for restoring hair growth in the future.

1. Sato, N., Leopold, P.L., and Crystal, R.G 1999. Induction of the hair growth phase in postnatal mice by localized, transient expression of Sonic hedgehog. J. Clin. Invest. 104:855-864.

2. Cotsarelis, G., Sun, T.T., and Lavker, R.M. 1990 Label-retaining cells reside in the bulge area of pilosebaceous unit: implications for follicular stem cells, hair cycle, and skin carcinogenesis. Cell. 61:1329-1337.

3. Lyle, S., et al. 1998. The C8/144B monoclonal antibody recognizes cytokeratin 15 and defines the location of human hair follicle stem cells. $J$ Cell. Sci. 111:3179-3188.

4. Stenn, K., Parimoo, S., and Prouty, S.M. 1998. Growth of the hair follicle: a cycling and regenerating biological system. In Molecular basis of epithelial appendage morphogenesis. C.M. Chuong, editor. R.G. Landes. Austin, TX. 111-130.

5. Saitoh, M., Uzuka, M., and Sakamoto, M. 1970 Human hair cycle. J. Invest. Dermatol. 54:65-81.

6. Reynolds, A.J., and Jahoda, C.A. 1992. Cultured dermal papilla cells induce follicle formation and hair growth by transdifferentiation of an adult epidermis. Development. 115:587-593.

7. Hardy, M.H. 1992. The secret life of the hair follicle. Trends Genet. 8:55-61.

8. Chuong, C.M., editor. 1998. Molecular basis of epithelial appendage morphogenesis. R.G. Landes. Austin, TX. 449 Pp.

9. Millar, S. 1997. The role of patterning genes in epidermal differentiation. In Cytoskeletal-membrane interactions and signal transduction. P. Cowin and M.W Klymkowsky, editors. R.G. Landes. Austin, TX. 87-102.

10. Oro, A.E., and Scott, M.P. 1998. Splitting hairs: dissecting roles of signaling systems in epidermal development. Cell. 95:575-578.

11. Paus, R., and Cotsarelis, G. 1999. The biology of hair follicles. N. Engl. J. Med. 341:491-497.

12. Gat, U., DasGupta, R., Degenstein, L., and Fuchs, E. 1998. De novo hair follicle morphogenesis and hair tumors in mice expressing a truncated beta-catenin in skin. Cell. 95:605-614.

13. Hammerschmidt, M., Brook, A., and McMahon, A.P. 1997. The world according to hedgehog. Trends Genet. 13:14-21.

14. Ming, J.E., Roessler, E., and Muenke, M. 1998. Human developmental disorders and the Sonic hedgehog pathway. Mol. Med. Today. 4:343-349.

15. Murone, M., Rosenthal, A., and de Sauvage, F.J. 1999. Sonic hedgehog signaling by the patched-smoothened receptor complex. Curr. Biol. 9:76-84.

16. Hahn, H., et al. 1996. Mutations of the human homolog of Drosophila patched in the nevoid basal cell carcinoma syndrome. Cell. 85:841-851.

17. Johnson, R.L., et al. 1996. Human homolog of patched, a candidate gene for the basal cell nevus syndrome. Science. 272:1668-1671.

18. Bitgood, M.J., and McMahon, A.P. 1995. Hedgehog and Bmp genes are coexpressed at many diverse sites of cell-cell interaction in the mouse embryo. Dev. Biol. 172:126-138.

19. Iseki, S., et al. 1996. Sonic hedgehog is expressed in epithelial cells during development of whisker, hair, and tooth. Biochem. Biophys. Res. Commun. 218:688-693.

20. St. Jacques, B., et al. 1998. Sonic hedgehog signaling is essential for hair development. Curr. Biol. 8:1058-1068.

21. Chiang, C., et al. 1999. Essential role for sonic hedgehog during hair follicle morphogenesis. Dev. Biol. 205:1-9.

22. Morgan, B.A., Orkin, R.W., Noramly, S., and Perez, A. 1998. Stage-specific effects of sonic hedgehog expression in the epidermis. Dev. Biol. 201:1-12.

23. Jahoda, C.A. 1998. Cellular and developmental aspects of androgenetic alopecia. Exp. Dermatol. 7:235-248. 